\title{
TEM Imaging of Edges and Point Defects in Monolayer Phosphorene
}

\author{
Yangjin Lee ${ }^{1}$, Sol Lee ${ }^{1}$, Jun-Yeong Yoon ${ }^{1}$, Jinwoo Cheon ${ }^{1}$, Hu Young Jeong ${ }^{2}$ and Kwanpyo Kim ${ }^{1}$ \\ ${ }^{1}$ Yonsei University, Seoul, Seoul-t'ukpyolsi, Republic of Korea, ${ }^{2}$ Ulsan National Institute of Science and \\ Technology (UNIST), Ulsan, Ulsan-gwangyoksi, Republic of Korea
}

Phosphorene, a single layer of black phosphorus (BP), has attracted intense research activity due to its unique properties, such as tunable band-gap and high charge carrier mobility, and exotic in-plane anisotropic physical properties [1]. However, transmission electron microscopy (TEM) imaging of atomic-scale defects of monolayer phosphorene, including point defects and edge configurations, has been elusive due to its degradation under ambient exposure as well as electron-beam-irradiation. Here, we demonstrate that phosphorene protected by graphene is an ideal sample geometry, which allows for reliable imaging of atomic-scale defects of phosphorene.

Bilayer-phosphorene/graphene vertical heterostructures were prepared inside a $\mathrm{N}_{2}$-filled globe-box via a dry transfer method, which allows for the fabrication of pristine samples without exposure to air. The optical transmittance of exfoliated phosphorene flakes on a transparent substrate (PDMS) was measured [2] as shown in Figure 1(a). We can confirm the layer thickness of phosphorene in a pristine condition, as the thickness of phosphorene samples was already correlated with the optical transmittance [2,3]. Preidentified BP flakes and graphene flakes were transferred to a $\mathrm{Si}_{3} \mathrm{~N}_{4} \mathrm{TEM}$ grid using dry transfer method and the vertical heterostructures were fabricated as shown in Figure 1(b). TEM image of bilayerphosphorene/graphene sample clearly shows the high crystallinity without noticeable structure deformation or residues as shown in Figure 1(c).

We observed that graphene can reduce e-beam-induced damages to nearby phosphorene layers in our recent study, [3]. In addition, graphene protection from one-side can induce the controlled thinning of phosphorene samples. For example, a bilayer phosphorene sample can be thinned down to a monolayer phosphorene using the controlled thinning process. The atomic resolution images of phosphorene were compared with TEM imaging simulation, confirming the layer-number-dependent phase contrast [3].

The atomic-scale edges configurations and point defects in monolayer phosphorene were investigated in detail. We found that the exposed phosphorene edges were predominantly aligned along the zigzag or (101) terminations. We also recorded the variation of edge population over time and studied the dynamical evolution as shown in Figure 2(a). The average fraction of the zigzag, (101), and armchair orientations were $48.3 \%, 42.1 \%$, and $9.6 \%$, respectively, as shown in Figure 2(b). This observation can be attributed to edge kinetics under e-beam-induced sputtering process. Monte Carlo simulations were utilized to reproduce the observed preferred edge formation process as shown in Figure $2 \mathrm{c}$ and $2 \mathrm{~d}$. The various point defects (mono-vacancy and di-vacancy) and extended defect structures in monolayer phosphorene were also observed and compared to theoretically-calculated structures [4]. 

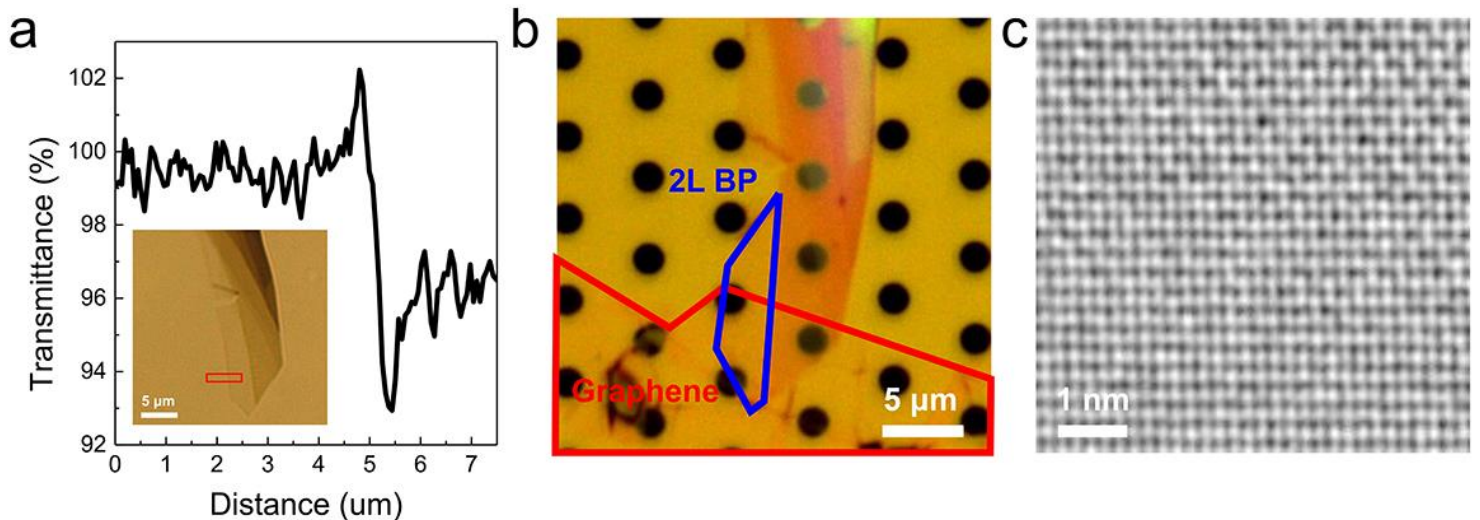

Figure 1. (a) Optical transmittance along the red box in inset. Inset: optical image of a bilayer phosphorene sample on PDMS. (b) Optical image of a bilayer-phosphorene/graphene vertical heterostructure on a Si3N4 TEM grid. (c) HR-TEM image of the bilayer phosphorene.
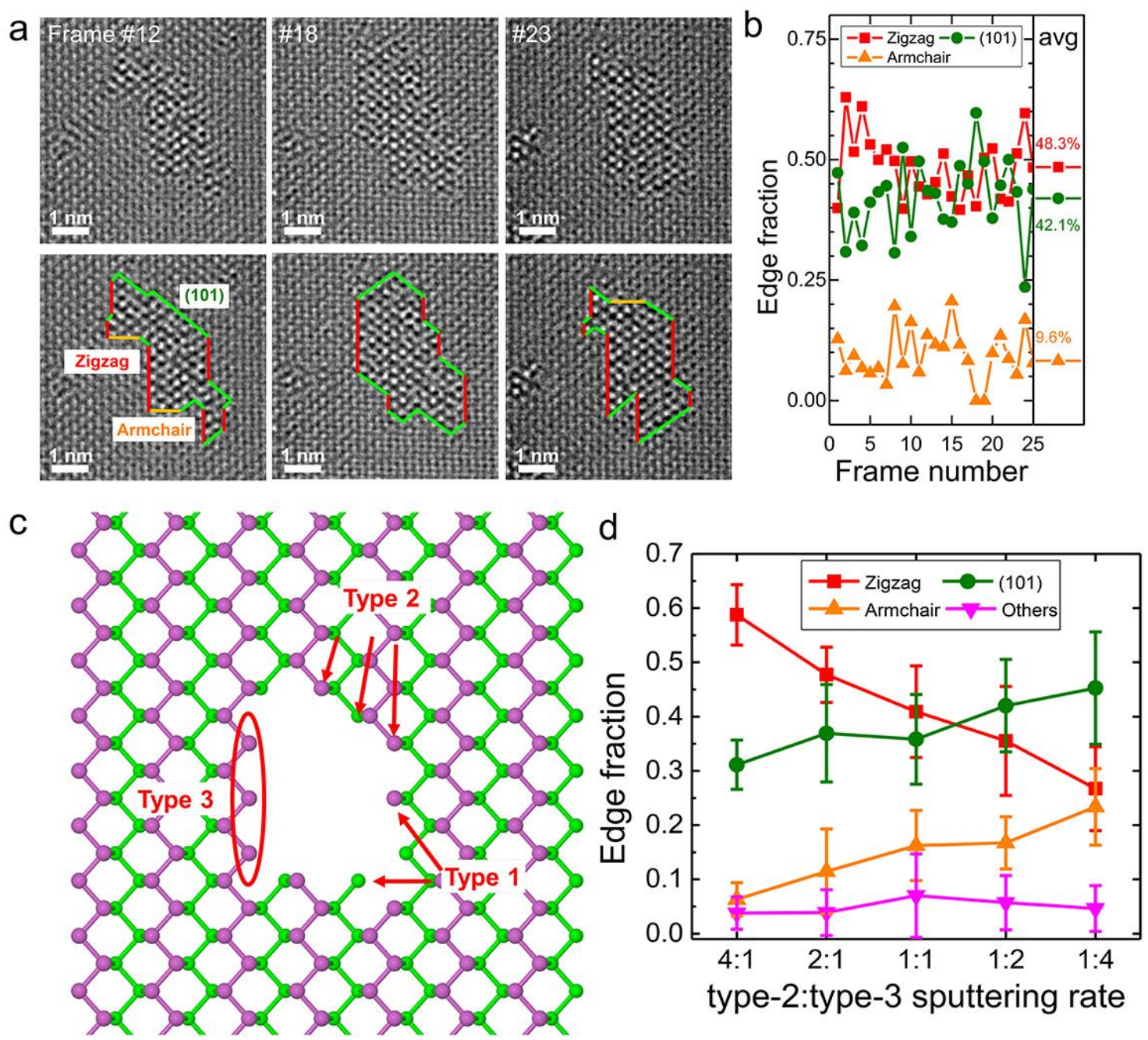

Figure 2. (a) Selected snapshots from TEM time series of a monolayer domain in bilayer surrounding. (b) Time-evolution of observed edge fraction from the time series. (c) Monte Carlo simulations for atom removal from exposed edge. Categorization of edge atoms depending on bond types. Type-1 atoms 
possess single neighbor atom. Type-2 edge atoms have one neighbor in the same atomic plane and one in the difference atomic plane. Type-3 edge atoms have two neighbors in the sample atomic plane. (d) Edge termination fraction as a function of sputtering probability ratio between type- 2 and type- 3 atoms.

\section{References}

[1] X. Ling et al., Proc. Natl. Acad. Sci. U. S. A. 112 (2015) 4523.

[2] Y. Lee et al., J Phys. D. J. Phys. D: Appl. Phys. 50 (2017) 084003.

[3] Y. Lee et al., Nano Lett. 20 (2020) 559-566.

[4] The authors acknowledge funding from the Basic Science Research Program through the National Research Foundation of Korea (NRF) funded by the Ministry of Education (NRF-2017R1A5A1014862, NRF-2018R1A2B6008104) and the Institute for Basic Science (IBS-R026-D1). 